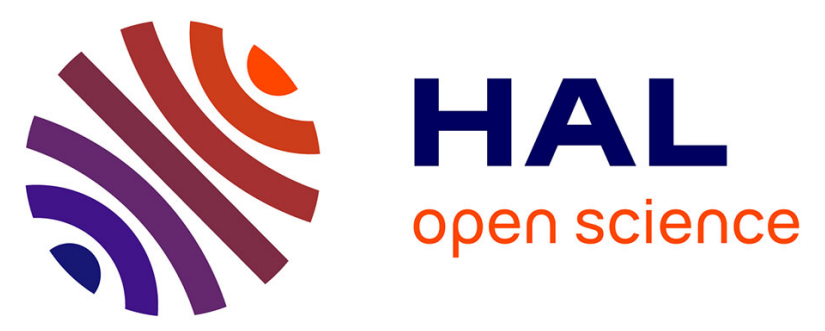

\title{
Upscaling of a dual-permeability Monte Carlo simulation model for contaminant transport in fractured networks by genetic algorithm parameter identification
}

F. Cadini, J. de Sanctis, I. Bertoli, Enrico Zio

\section{- To cite this version:}

F. Cadini, J. de Sanctis, I. Bertoli, Enrico Zio. Upscaling of a dual-permeability Monte Carlo simulation model for contaminant transport in fractured networks by genetic algorithm parameter identification. Stochastic Environmental Research and Risk Assessment, 2013, 27 (2), pp.505-516. 10.1007/s00477-012-0595-8 . hal-00934506

\section{HAL Id: hal-00934506}

https://hal-centralesupelec.archives-ouvertes.fr/hal-00934506

Submitted on 22 Jan 2014

HAL is a multi-disciplinary open access archive for the deposit and dissemination of scientific research documents, whether they are published or not. The documents may come from teaching and research institutions in France or abroad, or from public or private research centers.
L'archive ouverte pluridisciplinaire HAL, est destinée au dépôt et à la diffusion de documents scientifiques de niveau recherche, publiés ou non, émanant des établissements d'enseignement et de recherche français ou étrangers, des laboratoires publics ou privés. 


\title{
UPSCALING OF A DUAL-PERMEABILITY MONTE CARLO SIMULATION MODEL FOR CONTAMINANT TRANSPORT IN FRACTURED NETWORKS BY GENETIC ALGORITHM PARAMETER IDENTIFICATION
}

\author{
F. Cadini ${ }^{*}$, J. De Sanctis ${ }^{1}$, I. Bertoli ${ }^{1}$, E. Zio ${ }^{1,2}$ \\ ${ }^{1}$ Dipartimento di Energia - Politecnico di Milano \\ Via Ponzio 34/3, I-20133 Milan, Italy \\ *Corresponding author: francesco.cadini@ polimi.it; ph: +390223996355; fax: +390223996309 \\ ${ }^{2}$ Chair on Systems Science and the Energetic challenge, \\ European Foundation for New Energy- Électricité de France \\ Ecole Centrale Paris and Supelec
}

\begin{abstract}
The transport of radionuclides in fractured media plays a fundamental role in determining the level of risk offered by a radioactive waste repository in terms of expected doses. Discrete Fracture Networks (DFN) methods can provide detailed solutions to the problem of modeling the contaminant transport in fractured media. However, within the framework of the performance assessment (PA) of radioactive waste repositories, the computational efforts required are not compatible with the repeated calculations that need to be performed for the probabilistic uncertainty and sensitivity analyses of PA. In this paper, we present a novel upscaling approach, which consists in computing the detailed numerical fractured flow and transport solutions on a small scale and use the results to derive the equivalent continuum parameters of a lean, one-dimensional Dual-Permeability, Monte Carlo Simulation (DPMCS) model by means of a Genetic Algorithm search. The proposed upscaling procedure is illustrated with reference to a realistic case study of ${ }^{239} \mathrm{Pu}$ migration taken from literature.
\end{abstract}

Keywords: Radioactive waste repository; performance assessment; fracture networks; Upscaling; Monte Carlo simulation; genetic algorithms. 


\section{INTRODUCTION}

The modeling of solute transport through networks of fractures is of extreme importance for the risk assessment of radioactive waste repositories [1][2][3][4][5].

Multiple-continuum models, based on advection-dispersion equations, allow to approximately describe the transport phenomena on large scales but may fail to capture certain non-Fickian features on small scales. [6][7]. These models assume uniform dispersion, which is not always satisfied in fractured networks where flow and transport can take place in highly localized channels [6][7] and may even give rise to apparent scale dependence of the dispersivity [6][7][8][9].

Single-continuum models based on equivalent parameters may in general be exploited in case of very regular and well connected fracture networks, which can be approximated by a porous medium; however, significant biases may be introduced when more realistic fractures geometries are taken into account [10].

A detailed characterization and mapping of the fractured fields is usually not feasible due to the high degree of complexity and heterogeneity of the network, and information on geometric and conducting properties can be obtained only in terms of probability distributions and correlation functions [6][7][10][11][5].

Discrete Fracture Network (DFN) methods allow handling the high degree of complexity and heterogeneity of the networks of fractures [12][13]. These methods do not rely on volume averaging techniques, but solve the flow and transport problem in individual fractures by accounting for both anisotropy and stochasticity [13][14]. Nevertheless, an important drawback of these methods is related to the high degree of complexity of the modeling which requires an intensive computational effort and thus limits their range of applicability to near-field scales [6][7][14][5], and actually prevents the use in the probabilistic uncertainty and sensitivity analyses required in the PA of radioactive waste repositories.

Another approach proposes a stochastic continuum model which deterministically incorporates the major fracture planes and resorts to multiple statistical populations to model the statistical distribution of the hydraulic conductivity [4]; the model has been successfully applied to a threedimensional flow and transport problem in a fractured domain of the order of tens of meters, at the expense, again, of very large computational times and numerical problems due to the solution of the groundwater flow over a large domain in presence of very steep conductivity gradients.

An approach recently proposed to overcome these problems [15] is the "hybrid approach" [7]. It consists in carrying out detailed flow and transport simulations over small domains, thus limiting 
the numerical flow solution problems, and using the results to determine equivalent properties for continuum models to be used on larger scales [6][7][15][16]. When adopting this "upscaling" approach, the underlying assumption implicitly made is that the small domain is representative of the properties of the network and the results can be employed to upscale the conducting properties, i.e. to extend them to larger domains [6][7].

In this paper, an upscaling procedure is developed to determine the equivalent parameters of a lean, one-dimensional Dual-Permeability, Monte Carlo Simulation (DPMCS) model [17][18][19] of radionuclide migration at the field scale. Monte Carlo simulation is adopted because of its flexibility, necessary to account for the complex phenomena and geometries typically encountered in realistic Performance Assessments (PAs) [20][21]. On the other hand, the model onedimensionality is chosen in view of it utilization for fast dose predictions, within computationally demanding sensitivity and uncertainty analyses.

Specified probability distributions of fracture characteristic parameters are taken as input and numerical experiments are performed to collect reference data for the parameter calibration procedure. Different realizations of fracture fields are generated on small sub-domains by random sampling from the assigned distributions and by mapping into continuum grids with constant cell size, according to a recently suggested methodology [5][22]. Then, the MODFLOW and MT3DMS solvers [23][24] are employed to get detailed flow and transport solutions over these sub-domains [5]. These are used to compute a mean breakthrough curve which serves as a reference for the identification of the equivalent parameters of the DPMCS model. The parameters identification is performed by a Genetic Algorithm search aimed at minimizing the mean squared error between the DPMCS estimated breakthrough curve and the mean one of reference. Once the DPMCS model parameters are identified, they can be implemented in a new DPMCS model upscaled to a larger domain, as demonstrated in the application concerning ${ }^{239} \mathrm{Pu}$ migration in a fractured medium.

The paper is organized as follows. In Section 2 the parameter identification strategy and the resulting upscaling procedure are illustrated with reference to the DPMCS model, whose principal features are recalled. The results of the application of the procedure to the PA problem under consideration are reported in Section 3. Conclusions on the capabilities of the proposed method are drawn in Section 4. 


\section{GENETIC ALGORITHM-BASED UPSCALING PROCEDURE OF A DPMCS MODEL FOR TRANSPORT IN FRACTURE NETWORKS}

Fracture networks do not in general behave like a continuum [13]: channeling of flow and other phenomena may occur, which cannot be accounted for in continuum models. Only when the density and the connectivity of the network are large enough, equivalent descriptions can be found. In this case each fracture, if connected to the others, contributes to the overall permeability of the network; however, this is not simply the sum of the permeability of each individual fracture [8][13][25]. Moreover, the high degree of spatial variation of the conductivity of the fractures influences the velocity field, which in turn causes an increment in the spreading of the dissolved contaminants. This phenomenon causes an apparent increase of the dispersivity [8], which can even be found to vary with the observed scale [8][9]. It is therefore clear that even if an equivalent description is possible, the evaluation of the equivalent parameters of the network to solve flow and transport problems is far from being trivial. In certain cases, analytic expressions for equivalent properties can be obtained by using equivalent media and percolation theories [25][26][27], but the extension of these methods to realistic networks, whose parameters are described by realistic probability distributions, is burdensome. In such cases, if the network is not poorly connected, it is possible to resort to upscaling procedures which consist in carrying out detailed simulation of contaminants migration over small fractured sub-domains, assumed to be representative of the global properties of the network, and from these results determine the parameters for the equivalent continuum problem [6][7].

In this work, an upscaling procedure is developed in three main steps: $i$ ) a set of fracture networks is generated in a small sub-domain, by random sampling the characteristic parameters of each individual fracture, i.e. position, orientation, length and conductivity, from the corresponding distributions; these parameters are shown to be sufficient to determine a fracture network whose geometry is geometrically similar to that observed [10][11]; ii) each fracture network is mapped into continuum grids with constant cell size [5] and the corresponding flow and transport problems are solved by a detailed numerical model; and iii) the parameters of the DPMCS model are identified on the basis of the results of the detailed numerical model. The DPMCS model with the identified parameter values previously identified can then be applied to a larger, fractured domain. In what follows, the details of the upscaling procedure are provided with reference to the migration of a single species of non-reactive contaminant, for simplicity. 


\section{Fracture network generation}

Assuming that the individual properties are not correlated [22], fractures are generated in two groups, of fixed orientation $\left(\vartheta_{1}, \vartheta_{2}\right)$, with position uniformly distributed in a two-dimensional domain, and length $\left(L_{f}\right)$ and conductivity $\left(K_{f}\right)$ distributed according to power laws, such as:

$$
\begin{gathered}
P\left(L_{f} \geq l_{f}\right)=w_{L_{f}} l_{f}^{-a_{L_{f}}} \\
P\left(K_{f} \geq k_{f}\right)=w_{K_{f}} k_{f}^{-a_{K_{f}}}
\end{gathered}
$$

where $w_{L_{f}}$ and $w_{K_{f}}$ are normalization coefficients, and $a_{L_{f}}$ and $a_{K_{f}}$ are empirical parameters whose values are found to range between 1 and 3 for $a_{L_{f}}$ and around 0.4 for $a_{K_{f}}$ [22].

In order to limit the fracture spatial densities in the domain, the maximum density is controlled to decide whether the realization can be used or not. By choosing a small power law exponent for the fracture length distribution, the following criterion can be adopted to control maximum spatial density [22]:

$$
\frac{1}{A} \sum_{i=1}^{N_{f}} l_{f, i}<2.5
$$

where $A$ is the area of the domain, $l_{f, i}$ is the length of the $i-t h$ fracture and $N$ is the total number of fractures in the domain.

The lower limit is not computed by evaluating the percolation threshold; rather, here, in analogy with [22], it is instead determined indirectly by carrying out trial numerical simulations on the flow field in order to determine a value which guarantees a certain degree of connectivity and allows for a flow in the fractures from one side of the domain to the other.

According to the distributions and the constraint described above, a total number of $M_{f r}$ twodimensional fracture network realizations is generated by random sampling.

Flow and transport solution in a fractured sub-domain by MODFLOW and MT3DMS 
The non-reactive single species flow and transport problems are solved for each $M_{f r}$ fractured network realization by means of the finite element solvers MODFLOW and MT3DMS [23][24]. The corresponding rates of release outside the domain (i.e. the breakthrough curves) are subsequently averaged to obtain a mean breakthrough curve, whose the shape ultimately depends on the statistical properties assumed for the fracture field. An analysis is performed to assess whether the number of field realizations $M_{f r}$ guarantees the convergence of the mean of the breakthrough curve.

\section{Parameter identification and upscaling}

The mean breakthrough curve is used as reference for the identification of the equivalent flow and transport parameters of a continuum model. In this case, a DPMCS model is employed [19], whose main features are summarized as follows. In general the model can be one-, two- or threedimensional but here, for simplicity of illustration and without loss of generality, a one-dimensional spatial representation of the migration domain along the horizontal direction is considered.

The domain along which the transport occurs is subdivided in $N_{x}$ discrete zones, $x=1,2, \ldots, N_{x}$. The $N_{x}+1-t h$ compartment is an absorbing state representing the "environment". In each zone the amount of contaminant in the porous matrix and in the fracture system is to be determined. To this aim two categories of particles are introduced: the solutons, i.e. the particles of contaminant in the porous matrix, and the fracturons, i.e. the particles of contaminant travelling in the fracture system. In an elementary time interval $d t$, each one of these two particles can either travel to one of the neighboring zones, $x+1$ or $x-1$, with transition rates $f_{s}, f_{f}$ (forward) and $b_{s}, b_{f}$ (backward) respectively, or undergo a transition to the other domain in the same zone $x$, thus transforming into the other particle with corresponding rate $r_{s \rightarrow f, x}$ (for a soluton becoming a fracturon) or $r_{f \rightarrow s, x}$ (for a fracturon becoming a soluton). Finally, each contaminant particle might undergo a radioactive decay or a chemical reaction with rate $\lambda$. For simplicity of illustration, we assume that the daughter of the decay or of the chemical reaction is neither radioactive nor contaminant, so that it is of no further interest in the analysis.

By assuming that the process is $i$ ) linear (i.e. each particle is independent from the others), $i i$ ) markovian (i.e. the future state depends only on the present one) and by exploiting the properties of the probability generating functions (pgfs), a system of $2\left(N_{x}+1\right)$ coupled Kolmogorov's equations 
for the expected values of the numbers of solutons $(\mathrm{S})$ and fracturons $(\mathrm{F})$ at time $t$ in each zone $x=1,2, \ldots, \quad N_{x}+1$ can be obtained [17]:

$$
\begin{aligned}
& \frac{\partial S\left(x^{\prime}, t \mid 1_{s, x}, 0\right)}{\partial t}=-\left[b_{s}+f_{s}+l_{s} \rightarrow f, x^{\prime}+\lambda\right] S\left(x^{\prime}, t \mid 1_{s, x}, 0\right)+b_{s} S\left(x^{\prime}+1, t \mid 1_{s, x}, 0\right)+ \\
& +f_{s} S\left(x^{\prime}-1 \mid 1_{s, x}, 0\right)+r_{f \rightarrow s, x^{\prime}} F\left(z^{\prime}, t \mid 1_{s, x}, 0\right) \\
& \frac{\partial F\left(x^{\prime}, t \mid 1_{s, x}, 0\right)}{\partial t}=-\left[b_{f}+f_{f}+l_{f} \rightarrow s, x+\lambda\right] F\left(x^{\prime}, t \mid 1_{s, x}, 0\right)+b_{f} F\left(x^{\prime}+1, t \mid 1_{s, x}, 0\right)+ \\
& +f_{f} F\left(x^{\prime}-1 \mid 1_{s, x}, 0\right)+r_{s \rightarrow f, x^{\prime}} S\left(x^{\prime}, t \mid 1_{s, x}, 0\right)
\end{aligned}
$$

with $x^{\prime}=1,2, \ldots, N_{x}$ and where we assume to have one soluton in zone $x$ at time $t=0\left(1_{s, x}\right)$. In analogy to [18], the transition rates of the Kolmogorov-Dmitriev model may be related to the advection-dispersion model parameters. In this case, however, two sets of properties are needed, one describing the matrix and the other the fractures system to alternatively determine the solutons and the fracturons transition rates. For this reason, an analogy can be drawn with the dual permeability advection-dispersion model [28][29][30][31] (see Appendix A for more details).

The stochastic process of radionuclide migration across domains modeled by the system of partial differential equations (3.a) and (3.b) represents a continuous-time Markov process [32], whose solution is the probability of finding the $m$-th contaminant particle in compartment $z^{\prime}$ at time $t$. To account for realistic conditions, e.g. to account for non-homogeneities in time and space [33] or non-linear phenomena [20][21], a solution by Monte Carlo simulation may be quite adequate, whereby the stochastic migration of a large number $M$ of contaminant particles in the domain are simulated by repeatedly sampling their births, from the release sources and their transitions across the medium compartments, from the proper probability density functions.

Let us denote by $X_{m}(t)$ the state variable representing the position at time $t$ of the $m$-th contaminant particle ( $m=s, f$ for a soluton or a fracturon, respectively) in the discretized domain, i.e., $X_{m}(t)=n$ implies that at time $t$ the $m$-th particle is in cell $n=1,2, \ldots ., N_{x}+1$. The random walk of the individual radionuclide is simulated either until $X_{m}(t)=N_{x}+1$, i.e. until it exits the domain to the "environment", that is an absorbing state from which the particles are not allowed to come back to the migration domain, or until $t \geq T$, i.e. its lifetime crosses the time horizon $T$ of the analysis. For computational purposes, the time horizon $T$ is discretized in $N_{t}$ equally spaced time instants, with time step $\Delta t$; two different counters, Count ${ }_{s}(n, k)$ and Count ${ }_{f}(n, k)$, are associated to 
each compartment $n=1,2, \ldots ., N_{x}+1$ and each discrete time $k=1,2, \ldots ., N_{t}$. During the simulation, a one is accumulated in the counters Count ${ }_{s}(n, k)$ and Count ${ }_{f}(n, k)$ if a soluton or fracturon, respectively, resides, during its random walk, in compartment $n$ at time $k$. At the end of the $M$ simulated random walks of the contaminant particles, the values accumulated in the counters allow estimating the time-dependent probabilities of cell occupation by a soluton or a fracturon, $P_{n, s}(t)$ and $P_{n, f}(t)$ respectively:

$$
\begin{gathered}
P_{n, s}(k) \cong \frac{\text { Count }_{s}(n, k)}{M} \\
P_{n, f}(k) \cong \frac{\text { Count }_{f}(n, k)}{M}
\end{gathered}
$$

The total probability that a contaminant particle occupies cell $n$ at time $k$ is:

$$
P_{n}(k) \cong \frac{\text { Count }_{s}(n, k)+\text { Count }_{f}(n, k)}{M}=\frac{\text { Count }(n, k)}{M}
$$

Similarly, the probability density function of the release into the environment (breakthrough curve), $p d f_{e n v}(k)$, can be estimated as:

$$
p d f_{e n v}(k) \cong \frac{\text { Count }\left(N_{x}+1, k\right)}{M \cdot \Delta t}
$$

As anticipated above, the aim is that of identifying the parameters of the DPMCS model so that it is capable of approximating the mean breakthrough curve obtained on the small sub-domain by the MODFLOW and MT3DMS suite. In particular, the DPMCS model parameters to be determined are: $i)$ the equivalent conductivity of the fractures network, $K_{e q}$, which allows determining the velocity in the equivalent-continuum fracture system, $v_{\text {eq }}$, from Darcy's equation; $\left.i i\right)$ the equivalent dispersion coefficient in the fractures, $\left.D_{e q} ; i i i\right)$ and the mass exchange parameters $\left(r_{f \rightarrow s, x}\right.$ and $r_{s \rightarrow f, x}$ ) describing the process of retardation due to solute interaction with the matrix. The values of these parameters allow computing the transition rates of the DPMCS model (see Appendix A for 
details). In general, the method allows considering advective transport also in the porous matrix, but, in this modeling, for simplicity and without loss of generality, this is neglected and only the retardation due to diffusion into the matrix pores is considered.

A Genetic Algorithm [34] search is performed to find the equivalent DPMCS model parameters. The objective guiding the search is to minimize the mean squared error between the breakthrough curve obtained by the DPMCS model for the small sub-domain and the mean curve obtained by the MODFLOW and MT3DMS solutions of the $M_{f r}$ fracture field realizations on the same small subdomain. The optimization procedure entails running the DPMCS model several times in correspondence of properly chosen sets of values for the inputs $K_{e q}, D_{e q}, r_{f \rightarrow s, x}$ and $r_{s \rightarrow f, x}$ and computing the associated mean squared error with respect to the mean output of the MODFLOW and MT3DMS.

The parameter values found are implemented in the upscaled DPMCS model, which can be applied to larger, fractured domains. An exemplary application of this procedure is described in the next Section.

\section{UPSCALING APPLICATION ON A REALISTIC CASE STUDY}

The proposed procedure is applied to a case study from literature [35] concerning the release of

${ }^{239} \mathrm{Pu}$ from a near surface repository, whose design concept was studied by ENEA [35][36] and has similarities with the currently operative disposal facility of El Cabril, Spain [37]. As the radionuclides exit the repository floor, they migrate through the unsaturated zone, whose most important effect is that of retarding the infiltration towards the saturated zone (refer to [38] for further details). Then, as in [36], radionuclide migration in the groundwater domain is described to occur within a highly fractured limestone layer exhibiting extensive karstic phenomena. In this work, the equivalent parameters of the fracture network are estimated by adopting the upscaling procedure described in Section 3. For the properties of the fractures, the distributions of equations (1.a) and (1.b) are assumed with the parameters reported in Table 1. The criterion of equation (2) is considered for the maximum spatial density. The corresponding networks are sparsely fractured domains dominated by a few long fractures connecting their boundaries [22]. Moreover, the following correction is applied to the sampled value of the conductivity in order to account for the 
"stair step" pattern of the velocity field, which causes an apparent decrease of the hydraulic gradient from cell to cell in MODFLOW [22]:

$$
K_{\text {MODFLW }}=K \cdot(\sin |\vartheta|+\cos |\vartheta|)
$$

Table 1: Stochastic parameters for the generation of the fracture fields

\begin{tabular}{|l|l|}
\hline Orientation & $\vartheta_{1}=\frac{8}{21} \pi, \vartheta_{2}=\frac{5}{7} \pi$ \\
\hline Length & $w_{L_{f}}=5, a_{L_{f}}=1.28$ \\
\hline Conductivity & $w_{K_{f}}=10^{-4}, a_{K_{f}}=0.4$ \\
\hline
\end{tabular}

Numerical experiments, consisting in the generation of many different realizations of a 2-D fracture field, are carried out to collect reference data to be used for determining the equivalent conducting properties for larger scales application. The size of the elementary domain, adopted to compute the upscaling parameters, is $50 \mathrm{~m} \times 50 \mathrm{~m}$, discretized into cells of $1 \mathrm{~m} \times 1 \mathrm{~m}$; the right and left boundaries are given constant hydraulic head, whereas the upper and lower boundaries are set to noflow condition. To avoid boundary effects, the source is placed 5 cells, i.e. $5 \mathrm{~m}$, from the upstream boundary and the breakthrough curve is computed 5 cells, i.e. $5 \mathrm{~m}$, before the downstream boundary. Therefore, the actual distance along the horizontal direction is $40 \mathrm{~m}$. A planar source is employed, whose length is set equal to $40 \mathrm{~m}$ in the vertical direction. These dimensions are comparable to those adopted in [6]. For simplicity's sake, in the determination of the equivalent parameters, the migration of a single species of a non-reactive tracer is considered, characterized by a retardation factor $R=1$ (no adsorptions). The transport is simulated over a period of $300 h$ and the injection is assumed to be impulsive, i.e. all the particles are released at time $t=0$. A total of $M_{f r}=100$ realizations of the fracture field are generated and, for each realization, the flow and transport are solved by MODFLOW and MT3DMS numerical methods. The hydrological parameters used for the simulations are summarized in Table 2.

Table 2: Hydrological parameters

\begin{tabular}{|l|l}
\hline Matrix conductivity & $K_{m}=3 \cdot 10^{-7} \mathrm{~m} / \mathrm{s}$
\end{tabular}




\begin{tabular}{|l|l|}
\hline Dispersivities & $\alpha_{L f}=\alpha_{L m}=1 \mathrm{~m} \quad \alpha_{T f}=\alpha_{T m}=10 \mathrm{~m}$ \\
\hline Effective porosity & $\phi_{m}=\phi_{f}=0.15$ \\
\hline Upstream boundary hydraulic head & $h_{u p}=300 \mathrm{~m}$ \\
\hline Downstream boundary hydraulic head & $h_{\text {down }}=290 \mathrm{~m}$ \\
\hline Molecular diffusion coefficients & $D_{m, f}=D_{m, m}=1 \cdot 10^{-9} \mathrm{~m}^{2} / \mathrm{s}$ \\
\hline
\end{tabular}

The mean breakthrough curve over the different realizations is computed, together with its $\pm 1 \sigma$ standard deviation bands (Figure 1, solid line). This curve is considered as a reference for the calibration of the parameters of a small scale DPMCS model, which will be later exploited for developing an upscaled DPMCS model.

To promote model simplicity for repeated use in PA probabilistic analyses, a one-dimensional model is considered. The parameters to be determined are, then, the equivalent conductivity of the network $K_{e q}$, the equivalent dispersion coefficient $D_{e q}$ and the absorption and desorption coefficients $r_{f \rightarrow s, x}$ and $r_{s \rightarrow f, x}$. A Genetic Algorithm search is performed to determine the optimal values of the parameters of the one-dimensional, DPMCS model. A constraint is set on Peclet's number to preserve the physics of the problem $\left(P e=\frac{u L}{D}\right.$ where $u$ is the velocity, $L$ is a characteristic length and $D$ is the dispersion coefficient): a substantial change in this parameter would, in fact, result in the passage from an essentially advective problem to a predominantly diffusive problem. Therefore, instead of optimizing both $K_{e q}$ and $D_{e q}$, the equivalent conductivity and the reciprocal of the Peclet's number are encoded in the first two genes of the GA. The value of $D_{e q}$ can, then, be indirectly computed from these two parameters:

$$
D_{e q}=\frac{K_{e q}}{\phi} \frac{\overline{d h}}{d x} \Delta x \cdot P e^{-1}
$$

where $\Delta x$ is the discretization length in the horizontal direction (set equal to $\Delta x=1 \mathrm{~m}$ ) and $\frac{\overline{d h}}{d x}$ is the average hydraulic gradient over the domain. As mentioned in Section 3, the transport in the matrix subsystem is neglected because of the low permeability value (see Table 2). 
The range of variability of each gene for the search is determined by computing the minimum and maximum values of the corresponding physical quantities over all the generated fracture field realizations. As these values vary more than one order of magnitude, a logarithmic search is performed for all parameters apart from the reciprocal of the Peclet's number, whose variation is kept linear. The search ranges are set equal to [-5, -2] for $\ln \left(K_{e q}\right),[0,15]$ for $P e^{-1},[-7,-2]$ for $\ln \left(r_{f \rightarrow s, z}\right)$ and $\ln \left(r_{f \rightarrow s, z}\right)$; the number of bits for their binary representation is 10. A population of 100 chromosomes evolving for 200 generations is employed.

The GA search recursively solves the Monte Carlo scheme of Section 2, where $M=10^{4}$ fracturon particles are injected in the first upstream compartment of a dual-permeability, one-dimensional array of $N_{x}=40$ compartments of equal width $\Delta x=1 \mathrm{~m}$, and simulated over a time horizon of $T=300 h$, divided into $N_{t}=100$ equal time steps of constant width $\Delta t=3 h$. At the end of every simulation, the computed breakthrough curve, i.e. $p d f(k)$ (see equation (6)) is compared with that obtained from the MODFLOW and MT3DMS simulations and the fitness parameter, i.e. the mean squared error, is computed. To enhance its variability, the fitness parameter is set in logarithmic scale.

The optimal curve found with the GA procedure, i.e. the one with the minimum mean squared error, is reported in Figure 1 (dashed line). For the small-scale DPMCS model, the optimal values of the parameters found by the GA search are: $K_{e q}=1.1 \cdot 10^{-4} \mathrm{~m} / \mathrm{s}, \quad D_{e q}=1.4 \cdot 10^{3} \mathrm{~m}^{2} / \mathrm{s}$, $r_{f \rightarrow s, x}=1.4 \cdot 10^{-5} s^{-1}$ and $r_{s \rightarrow f, x}=3.0 \cdot 10^{-6} s^{-1}$. The general behavior follows that of the mean curve obtained by MODFLOW and MT3DMS, lying well within the $\pm 1 \sigma$ bands. It shows good predictions for the peak's value and time, whereas it is less satisfactory for the tail of the curve. This is expected, because of the one-dimensionality of the DPMCS model. In fact, the shape of the peak predicted by the detailed MODFLOW and MT3DMS is mainly due to the fastest tracer particles which are transported by advection along the main fractures and rarely interact with the matrix nor change their fracture direction. To intuitively describe this process, we can assume that, in a sparsely fractured domain dominated by a few long fractures connecting its boundaries, as the one under consideration [22], the advective transport is driven by a single fracture, e.g. with orientation $\vartheta_{1}=\frac{8}{21} \pi$ and average hydraulic conductivity $\overline{K_{f}} \cong 1.1 \cdot 10^{-3} \mathrm{~m} / \mathrm{s}$; then, we can write the average time $\tau$ one of these particles takes to exit the domain boundary as: 


$$
\tau \cong \frac{l \cdot \tan \left(\vartheta_{1}\right)}{\overline{v_{f}}}=\frac{\phi l}{\overline{q_{f}}} \cdot \tan \left(\vartheta_{1}\right)=\phi l \cdot\left(\overline{K_{f} \frac{d h}{d x}} \cdot\right)^{-1} \tan \left(\vartheta_{1}\right) \cong 24 h
$$

which is very close to the actual peak time obtained with MODFLOW and MT3DMS. Since the advection of the tracer particles along a main, straight fracture is well represented by a onedimensional process, then the DPMCS model, which is intrinsically one-dimensional, is capable of satisfactorily capturing this behavior. On the other hand, the shape of the tail of the curve is due to hydrodynamic dispersion, both at the microscale of the pores and macroscale of the fracture network, and to matrix interaction. The DPMCS model satisfactorily represents the matrix interaction phenomena, as demonstrated by the rather flat tail mainly due to those particles which are trapped in the matrix and then slowly released (see [19]); on the contrary, the differences in the tails predicted by the two models is mainly due to hydrodynamic dispersion, which is an intrinsically two-dimensional phenomenon (three-dimensional in more general applications). The larger spread of the particle release in the MODFLOW and MT3DMS due to dispersion is more evident at earlier times when the advection process is dominant, giving rise to the initial underestimation before $150 \mathrm{~h}$; then, simple mass conservation considerations explain the successive overestimation. The equivalent dispersion coefficient $D_{e q}$ in the one-dimensional DPMCS model is capable of partially capturing this behavior, but cannot exactly reproduce it. However, DPMCS tests carried out with different values of $D_{e q}$, not reported here for brevity' sake, show that the tail can be better reproduced at the expense of a decrease in the model peak accuracy and an increase in the total mean squared error [19][35]. 


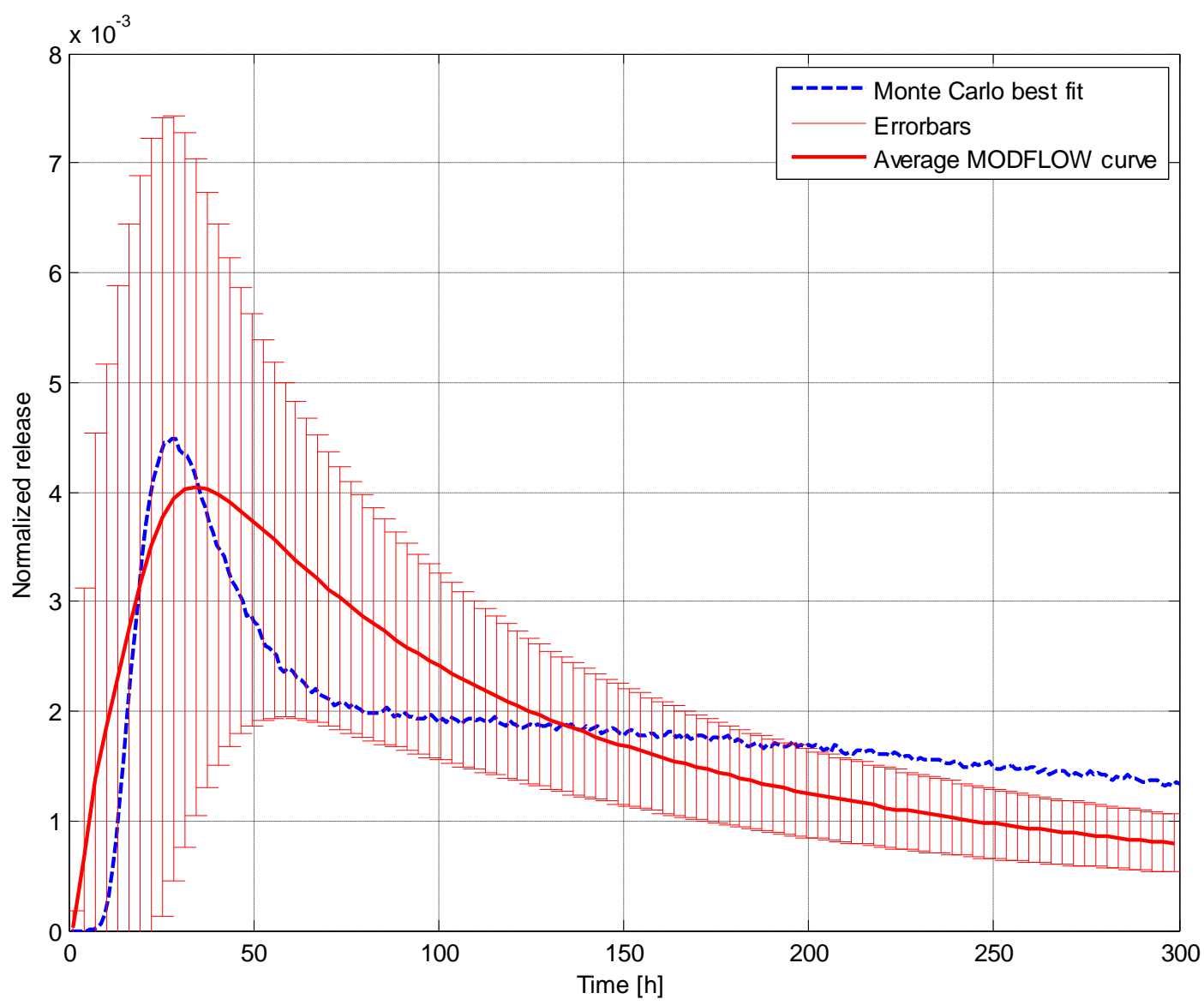

Figure 1: Comparison of MODFLOW and MT3DS mean breakthrough curve (solid line) and Monte Carlo curve (dashed line) on the $50 \mathrm{~m} \times 50 \mathrm{~m}$ domain

For demonstrating the upscaling capabilities, the simulation of ${ }^{239} \mathrm{Pu}$ migration in groundwater is carried out on a $200 \mathrm{~m}$ by $50 \mathrm{~m}$ fractured domain, generated with the input distributions of Section 3 and respecting the density criterion of equation (2). The same transport problem is solved both with MODFLOW and MT3DMS in two dimensions, whose results will be assumed as reference, and with a new, one-dimensional DPMCS model characterized by a different spatial discretization than the previous one but the same optimized values of the parameters.

In MODFLOW, a $40 \mathrm{~m}$ long planar source representing the repository is placed $5 \mathrm{~m}$ from the upstream boundary and the breakthrough curve is computed in the vicinity of the downstream boundary, at a distance of $190 \mathrm{~m}$. The ${ }^{239} \mathrm{Pu}$ particles are injected in the groundwater according to the release rate computed in [35] and the migration in the domain is assumed to occur under linear isothermal conditions with retardation factor equal to $R=5667$ [36]. Radioactive decay of ${ }^{239} P u$ is 
considered, with $\lambda_{239 P u}=2.876 \cdot 10^{-5} y^{-1}$ but, for simplicity's sake, the daughter of the decay is not further considered in the transport analysis. Both upstream and downstream boundaries are given constant hydraulic head with total difference on the domain $\Delta h=40 \mathrm{~m}$; the dispersivities are set to $\alpha_{L f}=\alpha_{L m}=10 \mathrm{~m}$ and $\alpha_{T f}=\alpha_{T m}=1 \mathrm{~m}$; all other hydrological parameters are left unchanged with respect to the previous case (Table 1). The spatial domain is discretized into $200 \times 50$ cells of $1 \mathrm{~m}$ by $1 \mathrm{~m}$, and the top and bottom boundaries are set as no-flow.

For the Monte Carlo simulation, a total of $M=10^{6}$ fracturon particles are injected in the first upstream compartment of a dual permeability, one-dimensional array of $N_{x}=19$ cells of equal length $\Delta x=10 \mathrm{~m}$, and their migration is simulated over a time horizon of $T=1000 \quad y$, divided into equal time steps of constant width $\Delta t=10 y$. The coarser spatial discretization employed for the Monte Carlo simulation is allowed by the equivalent parameters, which are valid at the scale of tens of meters.

In Figure 2, the breakthrough curve computed with the DPMCS model (dashed line) is compared with the reference one (solid line) obtained by averaging 10 simulations performed with MODFLOW and MT3DMS in correspondence of different fracture network realizations; the errorbars indicate the $\pm 1 \sigma$ ( $\sigma=$ standard deviation) variability of the results obtained with different networks. Overall satisfactory agreement is achieved: the peak height and time are well captured, as in the results of the calibration phase, since they are, again, mainly due to the one-dimensional average advective transport component. The shape of the curve is more symmetric than before, probably because the matrix interaction effects are "covered" by those due to dispersion, which is larger now due to the larger domain. The width of the curve is very similar to that of the reference one, which shows that $D_{e q}$ well describes the average longitudinal dispersion effects. On the other hand, the differences in the rising and descending parts of the curve are related to the asymmetry of the reference curve, which is the result of the intrinsic two-dimensional effects of the macroscopic dispersion at the full-scale domain due to the fractures. These effects cannot be captured unless a more complicated two dimensional DPMCS model is developed, but this is out of the scope of this work.

The apparent temporal upscaling is actually due to the fact that in the parameter calibration phase an instantaneous source of tracer particles with retardation factor $R=1$ (no adsorption) is employed, whereas in the full-scale simulation the more realistic ${ }^{239} P u$ source taken from [35] spreads 
approximately over a 1500 y time horizon; moreover, the ${ }^{239} \mathrm{Pu}$ retardation factor becomes $R=5.7 \cdot 10^{3}$, thus largely slowing down the transport process. Simulations carried out on the same full-scale domain with instantaneous source and $R=1$, not shown here for brevity, does not show significant differences in the model behavior, although the time scales become comparable to that of the calibration simulations.

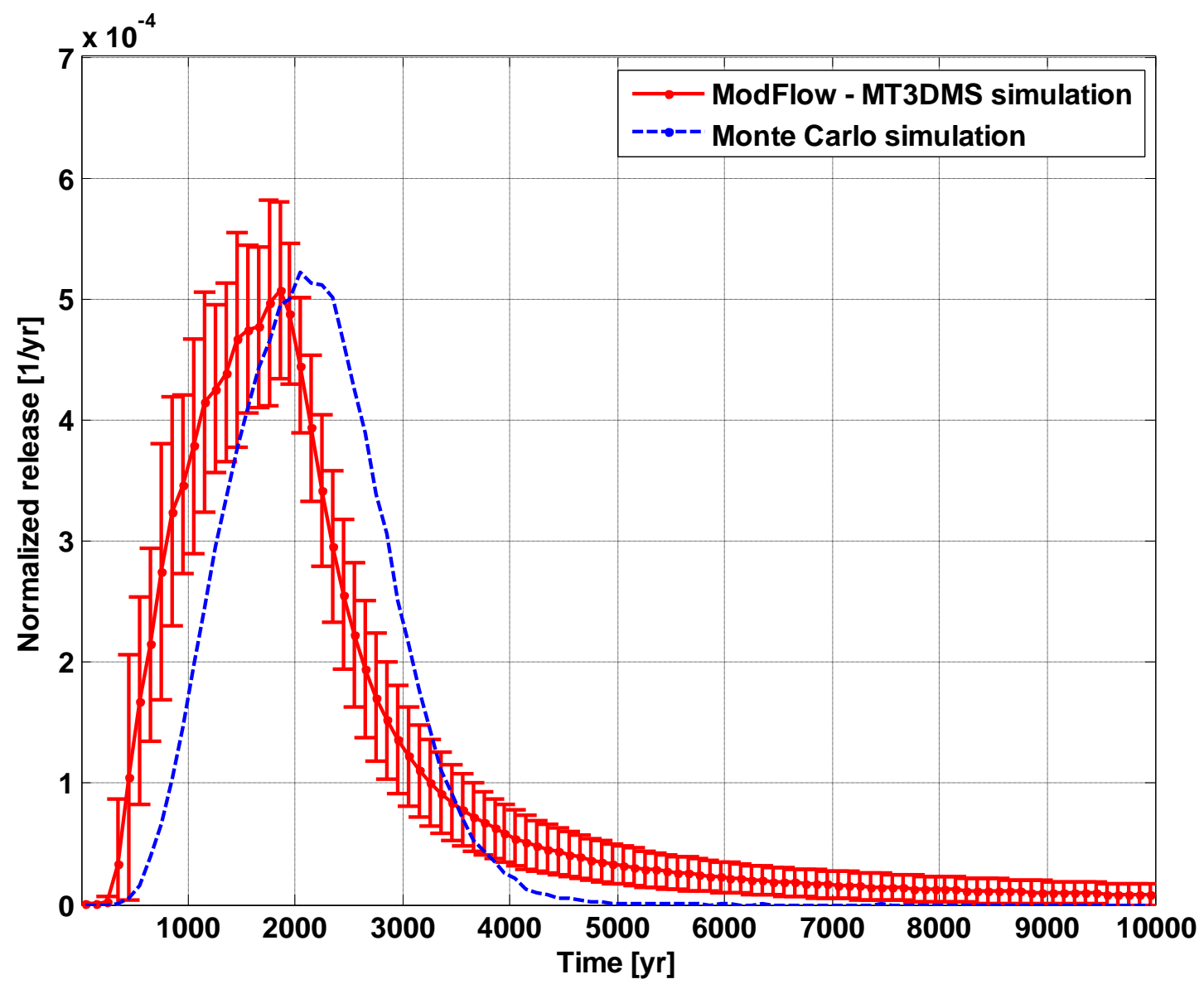

Figure 2: breakthrough curve at $200 \mathrm{~m}$ from the source: comparison between MODFLOW and MT3DMS (solid line) and DPMCS model (dashed line)

The entire procedure has been carried out with a 6GB RAM computer with an INTEL ${ }^{\circledR}$ CORETM i5 CPU M520 @ $2.40 \mathrm{GHz}$ processor. The calibration procedure requires a total of $1.17 \cdot 10^{4} s\left(1.392 \cdot 10^{3} s\right.$ for fracture field realization generation and computation of the mean curve, and $1.030 \cdot 10^{4} \mathrm{~s}$ to calibrate the parameters with the Genetic Algorithm). This procedure, however, needs to be performed only once and the results can subsequently be employed to solve the upscaled migration problem with a much faster and "leaner" model, which lends itself to be 
used for the repeated calculations of probabilistic uncertainty and sensitivity analysis of PA. In the case of the $200 m \times 50 m$ domain, for instance, it takes $5.24 \cdot 10^{3} s$ to compute the solution by the DPMCS model and $7.93 \cdot 10^{5} s$ by the MODFLOW and MT3DMS solver. Note that computational advantage increase as the domain becomes larger.

\section{CONCLUSIONS}

The assessment of the safety performance of a nuclear waste repository stands on the use of quantitative models for the prediction of the release and transport of radionuclides on extended time and spatial scales. In the transport process, fractures play a relevant role which must be properly evaluated and accounted for.

In this paper, we propose a novel approach for upscaling fracture networks properties into a continuum model. The approach consists in computing detailed solutions of the flow and transport problems in a two-dimensional sub-domain of the fractured medium with the finite difference solvers MODFLOW and MT3DMS, and using the results to determine the equivalent conducting properties to be employed in a one-dimensional Dual-Permeability, Monte Carlo Simulation (DPMCS) model. These properties are determined by means of a Genetic Algorithm search which minimizes the mean squared error between the breakthrough curve found with MODFLOW and MT3DMS and the one computed with the Monte Carlo scheme.

The approach is employed to carry out realistic simulations of ${ }^{239} \mathrm{Pu}$ release from a radioactive waste repository and migration within a fractured domain. By inserting the calibrated parameters in the Monte Carlo scheme, good agreement is found with the corresponding solution computed with a reference, detailed MODFLOW and MT3DMS simulation.

The proposed approach allows to drastically simplify the numerical model, by coarsening the spatial discretization and thus reducing the computational time. The procedure does require a certain effort but, once the equivalent parameters are found, they can be employed to solve transport problem on domains of any size, where the detailed solutions would become too expensive and even fail to reach convergence.

The choice of a one-dimensional model reduces the required computational times, so that the approach becomes attractive with respect to the fundamental PA tasks of uncertainty and sensitivity analyses on the physical parameters governing the radionuclides migration in groundwater. On the 
other hand, this choice affects the accuracy of the results, in particular due to the impossibility of fully capturing the two-dimensional (or, more generally, three-dimensional) dispersion patterns. A two-dimensional model would probably provide improved estimation accuracy. It is to be noted, however, that this would be at the cost of increased computational times, so that the model would not be effective from an uncertainty and sensitivity analyses viewpoint. Nonetheless, due to the flexibility offered by the Markovian modeling, investigations are currently being carried out in this regard.

\section{APPENDIX A}

In dual permeability and dual porosity models, the transport phenomenon is assumed to occur in two domains: the porous matrix and the fracture system. In particular, dual porosity models divide the medium in a mobile and an immobile region, assuming that flow occurs only in the larger permeability zone, whereas dual-permeability models assume the flow occurs in both subsystems. The model describes the heterogeneous porous media as a system made up of two different coupled subsystems and therefore the properties of the bulk medium depend on those of the different porous subsystems. Thus, following the notation of [30][31], the transport of solute in this system can be described by two coupled advection-reaction-dispersion equations, where the subscript $m$ refers to the matrix subsystem and $f$ to the fractures:

$$
\begin{gathered}
\frac{\partial\left(\theta_{f} R_{f} c_{f}\right)}{\partial t}=\nabla \cdot\left(\theta_{f} \mathbf{D}_{\mathbf{f}} \nabla c_{f}\right)-\nabla \cdot\left(\mathbf{q}_{\mathbf{f}} c_{f}\right)-\theta_{f} \lambda_{f} c_{f}-\frac{\Gamma_{s}}{w_{f}} \\
\frac{\partial\left(\theta_{m} R_{m} c_{m}\right)}{\partial t}=\nabla \cdot\left(\theta_{m} \mathbf{D}_{\mathbf{m}} \nabla c_{m}\right)-\nabla \cdot\left(\mathbf{q}_{\mathbf{m}} c_{m}\right)-\theta_{m} \lambda_{m} c_{m}-\frac{\Gamma_{s}}{w_{m}}
\end{gathered}
$$

where $\mathbf{D}$ is the dispersion tensor, $\lambda$ is the decay or chemical reaction term, $R$ is the retardation coefficient, $w$ is the relative volume of the pore system $\left(w_{m}=1-w_{f}\right), \theta$ is the water content, $\mathbf{q}$ is the Darcy's velocity, $c$ is the solute concentration and $\Gamma_{s}$ is the exchange term between the porous matrix and the fracture system. According to [30][31], assuming that only the diffusive contribution 
to the exchange process is taken into account and that the flow field is at steady-state, the exchange term $\Gamma_{s}$ can be expressed as:

$$
\Gamma_{s}=\alpha_{s} w_{m} \theta_{m}\left(c_{f}-c_{m}\right)
$$

The coefficient $\alpha_{s}$ in equation (A2) is [30][31]:

$$
\alpha_{s}=\frac{\beta}{a^{2}} D_{a}
$$

where $D_{a}$ is the effective diffusion coefficient at the interface, $\beta$ is a semi-empirical geometric factor and $a$ is the characteristic half-width of the porous matrix. The terms in equations (4.a) and (4.b) are all referred to the relative volume of the corresponding subsystem, with the exception of $\Gamma_{s}$, which is defined as the mass of solute per unit volume of bulk soil per unit time [Gerke and van Genuchten 1993a]. For the general form of equations (A1.a), (A1.b) and (A2) the interested reader should refer to [30][31].

Then under the hypotheses of: $i)$ one-dimensional domain, ii) no retardation $(R=1)$ and iii) constant water contents $\left(\theta_{m}\right.$ and $\left.\theta_{f}\right)$, constant pore velocities $v_{m}=\frac{q_{m}}{\phi_{m}} v_{f}=\frac{q_{f}}{\phi_{f}}\left(\right.$ where $\phi_{m}$ and $\phi_{f}$ are the porosities of the matrix and the fractures system, respectively), constant dispersion coefficients $D_{m}$ and $D_{f}$ and constant reaction rates $\lambda_{m}=\lambda_{f}=\lambda$, it is possible to write equations (4.a) and (4.b) in terms of the concentrations referred to the total bulk volume $\left(C_{f}=\theta_{f} w_{f} c_{f}\right.$ and $\left.C_{m}=\theta_{m} w_{m} c_{m}\right)$ and, subsequently, discretize them in space by a centered Euler method:

$$
\begin{aligned}
\frac{\partial C_{f}}{\partial t}=- & v_{f} \frac{\left(C_{f}(x+1, t)-C_{f}(x-1, t)\right)}{\Delta x}+D_{f} \frac{\left(C_{f}(x+1, t)+C_{f}(x-1, t)-2 C_{f}(x, t)\right)}{\Delta x^{2}}- \\
& -\alpha_{s} \frac{\theta_{m} w_{m}}{\theta_{f} w_{f}} C_{f}(x, t)+\alpha_{s} C_{m}(x, t)-\lambda C_{f}(x, t) \\
\frac{\partial C_{m}}{\partial t}= & -v_{m} \frac{\left(C_{m}(x+1, t)-C_{m}(x-1, t)\right)}{\Delta x}+D_{m} \frac{\left(C_{m}(x+1, t)+C_{m}(x-1, t)-2 C_{m}(x, t)\right)}{\Delta x^{2}}+ \\
& +\alpha_{s} \frac{\theta_{m} w_{m}}{\theta_{f} w_{f}} C_{f}(x, t)-\alpha_{s} C_{m}(x, t)-\lambda C_{m}(x, t)
\end{aligned}
$$


These equations are formally identical to (3.a) and (3.b); thus, by comparing the two systems of equations it is possible to write the following relationships for the forward and backward rates of the solutons and the fracturons:

$$
\begin{aligned}
& f_{s}=\frac{D_{m}}{\Delta x^{2}}+\frac{v_{m}}{2 \Delta x} ; \quad f_{f}=\frac{D_{f}}{\Delta x^{2}}+\frac{v_{f}}{2 \Delta x} \\
& b_{s}=\frac{D_{m}}{\Delta x^{2}}-\frac{v_{m}}{2 \Delta x} ; \quad b_{f}=\frac{D_{f}}{\Delta x^{2}}-\frac{v_{f}}{2 \Delta x}
\end{aligned}
$$

Note that, since the backward transition rates $b_{s}$ and $b_{f}$ cannot be negative, equations (A5.a) and (A5.b) require an upper limit for the spatial discretization cell:

$$
\Delta x<\min \left[\frac{2 D_{m}}{v_{m}} ; \frac{2 D_{f}}{v_{f}}\right]
$$

Analogously to the forward and backward rates, the exchange rates can be expressed as:

$$
\begin{gathered}
r_{f \rightarrow s, x}=\alpha_{s} \frac{\theta_{m} w_{m}}{\theta_{f} w_{f}} \\
r_{s \rightarrow f, x}=\alpha_{s}
\end{gathered}
$$

The difference in the exchange rates depends mainly upon the different relative volume of each system: the larger the volume of the subsystem is, the less probable the transition to the other subsystem is.

\section{References}

[1] Carr J.R., Fractal characterization of joint surface roughness in welded tuff at Yucca Mountain, Nevada. In The 30th US Symposium on Rock Mechanics (USRMS), 1989

[2] Cvetkovic V., Painter S., Outters N., and Selroos J.O., Stochastic simulation of radionuclide migration in discretely fractured rock near the Äspö hard rock laboratory. Water Resour Res, 40(2):W02404, 2004 
[3] Hölttä P., Poteri A., Hakanen M., and Hautojärvi A., Fracture flow and radionuclide transport in block-scale laboratory experiments. Radiochimica Acta/International journal for chemical aspects of nuclearscience and technology, 92(9-11):775-779, 2004.

[4] Hendricks Franssen, H.J.W.M., Gomez-Hernandez, J.J., 3D inverse modelling of groundwater flow at a fractured site using a stochastic continuum model with multiple statistical populations, Stochastic Environmental Research and Risk Assessment, 16, 155-174, 2002.

[5] Reeves, D.M., Pohlmann, K.F., Pohll, G.M., Ye, M., Chapman, J.B., Incorporation of conceptual and parametric uncertainty into radionuclide flux estimates from a fractured granite rock mass, Stochastic Environmental Research and Risk Assessment, 24, 899-915, 2010.

[6] Benke, R. and Painter, S., 2003, Modelling conservative tracer transport in fracture networks with a hybrid approach based on the Boltzmann transport equation, Water Resour. Res., 39(11), 1324, doi:10.1029/2003WR001966, 2003.

[7] Painter, S. and Cvetkovic, V., Upscaling discrete fracture network simulations: An alternative to continuum models. Water Resour. Res., 41, W02002, doi:10.1029/2004WR003682, 2005.

[8] Neuman, S.P., Universal Scaling of Hydraulic Conductivities and Dispersivities in Geological Media, Water Resour. Res., 26(8), 1749-1758, 1990.

[9] Vanderborght, J., Kasteel, R., and Vereecken, H., Stochastic Continuum Transport Equations for Field-Scale Solute Transport: Overview of Theoretical and Experimental Results, Vadose Zone J., 5, 184-203, 2006

[10] Chevalier, S., Buès, M.A., Tournebize, J., Banton, O., Stochastic delineation of wellhead protection area in fractured aquifers and parametric sensitivity study, Stochastic Environmental Research and Risk Assessment, 15, 205-227, 2001.

[11] Long, J.C.S., Billaux D.M., From field data to fracture network modeling: an example incorporating spatial structure, Water Resources Research, 23(7), 1201-1217, 1987.

[12] L. Smith and F.W. Schwartz. An analysis of the influence of fracture geometry on mass transport in fractured media. Water Resour Res, 20(9):1241-1252, 1984.

[13] Long, J.C.S., Remer, J.S., Wilson, C, .R. and Witherspoon P.A. (1982), Porous Media Equivalents for Networks of Discontinuous Fractures, Water Resour. Res., 18(3), 645-658.

[14] Cacas M.C., Ledoux E., De Marsily G., Tillie B., Barbreau A., Durand E., Feuga B., and Peaudecerf P., Modeling fracture flow with a stochastic discrete fracture network: calibration and validation: 1. The flow model. Water Resour. Res., 26(3):479-489, 1990.

[15] National Research Council. Rock Fractures and Fluid Flow, Contemporary Understanding and Applications, 1996 
[16] Blum P., Mackay R., Riley M.S., Stochastic simulations of regional scale advective transport in fractured rock masses using block upscaled hydro-mechanical rock property data, J. Hydrol., $369,318-325,2009$.

[17] Marseguerra, M. and Zio, E., Modelling the Transport of Contaminants in Groundwater as a Branching Stochastic Process, Ann. Nucl. Energy, 24(8), 325-644, 1997.

[18] Ferrara A., Maseguerra M. and Zio E., A comparison between the advection-dispersion and the Komogorov-Dmitriev model for groundwater contaminant transport, Ann. Nucl. Energy, 26(12), 1083-1096, 1999.

[19] Cadini F., Bertoli I., De Sanctis J. and Zio E., Monte Carlo simulation of contaminant migration in a dual-permeability fractured medium, submitted to Water Resour. Res.

[20] Marseguerra, M., Patelli, E., and Zio, E., Groundwater contaminant transport in presence of colloids: a stochastic nonlinear model and parameter identification, Ann. Nucl. Energy , 28(8), 777803, 2001.

[21] Cadini, F., De Sanctis, J. and Zio E., "Challenges in Monte Carlo Simulation of Non-Linear Radioactive Contaminant Release Processes", Proceedings of the International High Level Radioactive Waste Conference, IHLRWC, Albuquerque, USA, April, 10-14, 2011.

[22] Reeves D.M., Benson D.A., and Meerschaert, M.M., Transport of conservative solutes in simulated fracture networks: 1. Synthetic data generation. Water Resour. Res., 44:W05404, 2008 [23] McDonald M.G. and Harbaugh A.W., A modular three-dimensional finite-difference groundwater flow model. Department of the Interior, Reston, VA (US), 1988

[24] Zheng C., Wang P.P., and ALABAMA UNIVERSITY. MT3DMS: a modular threedimensional multispecies transport model for simulation of advection, dispersion, and chemical reactions of contaminants in groundwater systems; documentation and user's guide. US Army Corps of Engineers, Engineer Research and Development Center, 1999.

[25] Hestir K. and Long J.C.S. (1990), Analytical Expressions for the Permeability of Random Two-Dimensional Poisson Fracture Networks Based on Regular Lattice Percolation and Equivalents Media Theories, J. Geophys. Res., 95(B13), 21,565-21,581.

[26] Sahimi M., Applications of Percolation Theory, Taylor \& Francis, London, 1994.

[27] Sahimi M., Flow and transport in porous media and fractured rock: from classical methods to modern approaches, VCH, Weinheim, 1995.

[28] Barenblatt, G.I., Zheltov, Iu. P. and Kochina, I. N., Basic concepts in the theory of seepage of homogeneous liquids in fissured rocks, J. Appl. Math. Mech., 24, 1286-1303, 1960. 
[29] Warren, J.E. and Root, P.J., The behavior of naturally fractured reservoirs, Soc. Petrol. Eng. J., 3, 1861-1874, 1985.

[30] Gerke, H.H. and van Genuchten, M.T., A Dual-Porosity Model for Simulating the Preferential Movement of Water and Soluted in Structured Porous Media, Water Resour. Res., 29(2),305-319, 1993.

[31] Gerke, H.H. and van Genuchten, M.T., Evaluation of a First-Order Water Transfer Term for Variably Saturated Dual-Porosity Flow Models, Water Resour. Res., 29(4), 1225-1238, 1993.

[32] Papoulis, A. and Pillai, S.U., Probability, random variables, and stochastic processes, Boston, McGraw-Hill, 2002.

[33] Dentz, M., Cortis, A., Scher, H. and Berkowitz, B., Time behavior of solute transport in heterogeneous media: Transition from anomalous to normal transport. Adv. Water Res., 27(2), 155173, 2004.

[34] D.E. Goldberg. Genetic algorithms in search, optimization, and machine learning. AddisonWesley, 1989.

[35] Cadini, F., De Sanctis, J., Girotti, T., Zio, E., Luce, A. and Taglioni, A., Monte Carlo estimation of radionuclide release at a repository scale. Ann. Nucl. Energy, 37:861-866, 2010

[36] Chapman, N.A., Preliminary Feasibility Assessment for Near-Surface Engineered LLW Repositories at Two Sites. Technical report, ENEA, 1997.

[37] Zuloaga, P., Andrade, C. and Saaltink, M.W. Long term water scenario in low-level waste disposal vaults, with particular regard to concrete structures in el cabril, cordoba, spain. In Journal de Physique IV (Proceedings), volume 136, pages 49-59, 2006

[38] Cadini, F., De Sanctis, J., Girotti, T., Zio, E., Luce, A., and Taglioni, A., Monte Carlo-based assessment of the safety performance of a radioactive waste repository. Rel. Eng. And Syst. Safety, 95,859-865, 2010. 